\title{
Short Periodicities in Latitudinal Variation of Sunspots
}

\author{
Bang-Yeop Kim ${ }^{1}$ and Heon-Young Chang ${ }^{2 \dagger}$
}

${ }^{1}$ Geostationary Satellite Control Department, Satellite Operations and Application Division, Korea Aerospace Research Institute, Daejeon 305-333, Korea

${ }^{2}$ Department of Astronomy and Atmospheric Sciences, Kyungpook National University, Daegu 702-701, Korea

The latitudinal variation of sunspots appearing during the period from 1874 to 2009 has been studied in terms of centerof-latitude (COL). The butterfly diagram has been used to study the evolution of the magnetic field and the dynamics at the bottom of the solar convection zone. Short-term periodicities have been of particular interest, in that they are somehow related to the structure and dynamics of the solar interior. We thus have focused our investigation on shortterm periodicities. We first calculated COL by averaging the latitude of sunspots with the weight function in area. Then, we analyzed the time series of COL using the wavelet transform technique. We found that a periodicity of $\sim 5$ years is the most dominant feature in the time series of COL, with the exception of the $\sim 11$ year solar cycle itself. This periodicity can be easily understood by considering small humps between the minima in the area-weighted butterfly diagram. However, we find that periodicities of $\sim 1.3(0.064), \sim 1.5(0.056)$, or $\sim 1.8(0.046)$ years ( $\left.\frac{1}{\text { month }}\right)$, which have been previously suggested as evidence of links between the changing structure of the sunspot zone and the tachocline rotation rate oscillations, are insignificant and inconsistent. We therefore conclude that the only existing short-term periodicity is of $\sim 5$ years, and that periodicities of $\sim 1.3, \sim 1.5$, or $\sim 1.8$ years are likely to be artifacts due to random noise of small sunspots.

Keywords: Sun, sunspots, data analysis

\section{INTRODUCTION}

The solar magnetic cycle can be studied in terms of the latitudinal variation of the location of sunspots with time (Antalova \& Gnevyshev 1965, 1983, Pelt et al. 2000, Temmer et al. 2002, 2006, Zolotova \& Ponyavin 2006, 2007, Donner \& Thiel 2007, Chang 2007, 2008, 2009, Ternullo 2007a, 2010a, Solanki et al. 2008, Goel \& Choudhuri 2009, Li et al. 2008, 2009a, b, 2010a, b, Zolotova et al. 2009, Li 2010). For instance, Tuominen (1952) noticed the existence of a difference in the meridional motions of spot groups during the odd and the even cycles, and suggested the existence of a 22-year periodicity in the solar meridional flow. Pulkkinen et al. (1999) also studied the long-term variation of sunspot latitudes, and reported a period of about 90 years. Moreover, they demonstrated an idea that the solar North-South asymmetry phenom- enon could be explained by the oscillation of a quadrupolar component of a solar-mixed parity mode magnetic field. In this way, the butterfly diagram may be used to study the solar activity, leading even to constraining a solar dynamo model (Waldmeier 1957, 1971, Li et al. 2002, Solanki et al. 2008).

Since sunspots are formed soon after the emergence of an active region and generally die long before the active region itself, they can be considered as tracers of freshly emerged magnetic flux. Thus, under the assumption that the sunspot butterfly diagram reflects the evolution of the magnetic field and the dynamics at the bottom of the solar convection zone, which has been shown as reasonable (Hathaway et al. 2003), the search for such a shortterm periodicity is intriguing, and calls for more careful analyses.

Recently, there have been some efforts to search for (c) This is an Open Access article distributed under the terms of the Creative Commons Attribution Non-Commercial License (http://creativecommons.org/licenses/by-nc/3.0/) which permits unrestricted non-commercial use, distribution, and reproduction in any medium, provided the original work is properly cited.
Received Apr 08, 2011 Revised Apr 18, 2011 Accepted Apr 22, 2011 ${ }^{\dagger}$ Corresponding Author

E-mail: hyc@knu.ac.kr

Tel: +82-53-950-6367 Fax: +82-53-950-6359 
short-term periodicities in the latitudinal distribution of sunspots (Krivova \& Solanki 2002, Knaack et al. 2005) in addition to long-term periodicities, such as $~ 11$-year (Schwabe 1843), 44-year (Javaraiah 2003), 53-year (Le \& Wang 2003), 78-year (Wolf 1862), 80-90-year (Gleissberg 1971), and $\sim 65-130$ year quasi-periodic cycles (Nagovitsyn 1997). Interestingly, these short-term periodicities have been related to the structure and dynamics of the solar interior, such as oscillations at $\sim 1.3$ years observed in the rotation rate at the base of the convection zone (Howe et al. 2000), or a torsional wave pattern with alternating latitude zones of slow and fast rotation (Howard \& LaBonte 1980). For instance, Krivova \& Solanki (2002) attempt to demonstrate that the periodicity of the tachocline rotation rate oscillations can actually be traced in the sunspot activity.

In this paper, we take the area of sunspots into account in the latitudinal solar-cyclic behavior of sunspots (Cho \& Chang 2011). This consideration is required, as it reduces the noise level of a power spectrum in the sense that small sunspots prevail in the butterfly diagram, which scatter over wider latitude ranges than larger ones. The butterfly diagram is quite noisy because all sunspots are given equal weight in the butterfly diagram, regardless of their temporal and spatial extension. For example, $65 \%$ of groups (the smallest ones) contribute to only $\approx 10 \%$ of the total spotted area, as was pointed out by Ternullo (2007b). Hence, periodical tests are apt to be biased by randomly scattered small sunspots unless the area of sunspots is properly managed. So far, this simple fact that the butterfly diagram appears to be noisy due to small sunspots has been ignored. For instance, Ternullo (2010a) has claimed that there is a fine structure in the butterfly diagram that evolves over time, and that the averaged latitude of sunspots apparently drifts alternatingly equatorward and poleward for short time intervals, i.e. $\sim 2$ years. He came to this conclusion through a visual inspection, without any periodogram analysis. More seriously, he averaged the latitudes of sunspots with a moving window, whose width is a decisive factor to the result, without taking care of the area of sunspots. We review the recently reported short-term periodicities in the butterfly diagram by replotting it by weighting the sunspot with its area (Krivova \& Solanki 2002, Ternullo 2007a, 2010a,b). By doing so, we may or may not confirm the short-term periodicities recently reported.

This paper begins with descriptions of how the centerof-latitude (COL) is defined, and of the wavelet analysis in Section 2. We present and discuss the results obtained with the technique of wavelet transform in Section 3. Fi- nally, we summarize and conclude in Section 4 .

\section{CENTER-OF-LATITUDE OF SUNSPOT AND WAVELET ANALYSIS}

The data used for analysis were the daily sunspot area and its latitude obtained from the National Aeronautics and Space Administration (NASA) website ${ }^{1}$. The data set we analyze in the current analysis spans from 1874 to 2009. Here, we consider the distribution of the COL. We collectively compute COL for sunspots appearing in both hemispheres by taking the absolute value of the latitude, which is given by

$$
\mathrm{COL}=\sum_{i}\left(A_{i} \times\left|L_{i}\right|\right) / \sum_{i} A_{i},
$$

where $A_{i}$ is the $i$-th sunspot's area and $L_{i}$ is its latitude. We have carried out the summation over a period of one calendar month. It should be noted that the collective COL is not identical to the average of COLs, due to the fact that some sunspots separately appear in only one of the northern or southern hemispheres.

In Fig. 1, we show the averaged COL as a function of time. The yearly averaged COL is also shown with a thick curve for comparison. Note, however, that the thick curve is given simply for clarity, and the thin curve alone is used in the analysis. The solar cycle begins when COL is high, and COL gradually decreases in general as the solar cycle proceeds. We note, however, that small humps (or short plateaus) appear around almost all solar maxima. That

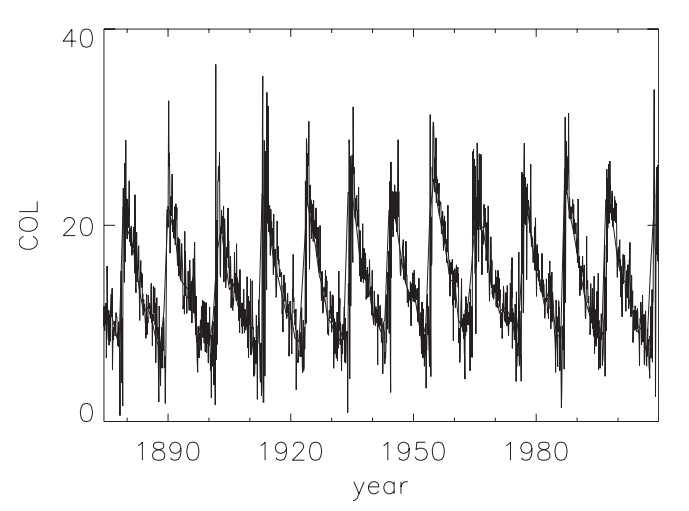

Fig. 1. The averaged center-of-latitude (COL) as a function of time from 1874 to 2009 . Thick and thin curves are yearly and monthly averaged $\mathrm{COL}$, respectively. Note, however, that the thick curve is given for a simple clarity and the thin curve alone is used in the analysis.

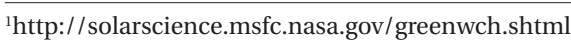


is, COL is not monotonically decreasing. A similar conclusion was drawn by Antalova \& Gnevyshev (1983), who stated that the 11-year cycle consists of the superposition of two maxima which peak at different times and different latitudes, and that the second peak occurs at a lower latitude than the first. Recently, Ternullo (2010b) has also shown that the sunspot has the tendency to preferentially appear in some photospheric regions tightly limited in latitude,givinga 'leopard skin' appearance to the butterfly diagram. This is in contrast to the traditional view that the average latitude of sunspots in the butterfly diagram steadily drifts equatorward. One may be interested in further pursuing this non-monotonic behavior to relate the latitudinal preference to postulating 'active latitude' suggested by Solanki et al. (2008).

In the present analysis, we employ the wavelet analysis technique associated with the Gabor transform. This method is sometimes referred to as time/frequency analysis. Compared to the Fourier transform FT, which is defined by

$$
F T(v)=\int S(t) \exp (-i 2 \pi v t) d t
$$

the Gabor transform GT is effectively defined as

$$
G T\left(v, t_{0}\right)=\int S(t) G\left(t-t_{0}\right) \exp \left[-i 2 \pi v\left(t-t_{0}\right)\right] d t
$$

where $S(t)$ is the signal as a function of time $t, v$ is the cyclic frequency, and $G(t)$ is the window function. In the above form, the wavelet transform $W T$ can be considered as a transform of a signal derived from an original wavelet by dilation in time, i.e., as

$$
W T\left(d, t_{0}\right)=\int S(t) W\left(\frac{t-t_{0}}{d}\right) d t,
$$

where $d$ is a dilation coefficient. Put simply, the wavelet transform is the correlation of the signal $S(t)$ and a running wavelet $W(t)$.

Several wavelet-forms can be selected, but in practice the most popular choice is the Morlet wavelet (Grossmann \& Morlet 1984). It is a sinusoidal wave with a Gaussian window:

$$
W\left(\frac{t}{d}\right)=\exp \left[-\pi\left(\frac{t}{d}\right)^{2}\right] \exp \left(-2 \pi i \frac{t}{d}\right)
$$

where $d$ is a dilation coefficient. Thus, it will provide information around time $t_{0}$ and around frequency $v=1 / d$. Its squared modulus is proportional to the power of the signal. This information is displayed in a two-dimensional plot, showing variations of the power with time and frequency. The shape of the window is an important parameter, asit will allow optimization in time and in frequency of the information extracted. In this paper, we adopt the Morlet wavelet transform throughout the analysis.

\section{RESULTS AND DISCUSSION}

In Fig. 2, as an example, we show a 2-dimensional power spectrum obtained by the wavelet analysis to demonstrate its robustness. As mentioned in Section 2, the power spectrum is shown in a 2-dimensional plot as a function of time in month and frequency in $\frac{1}{\text { month }}$. The data set covers 1628 months, from May in 1874 to December in 2009. The power is normalized with noise, and shown in an arbitrary unit. Not surprisingly, the main peak in the whole power spectrum occurs where the frequency is $\sim 0.0078 \frac{1}{\text { month }}$, whose period corresponds to $\sim 10.68$ years. In Fig. 3 , we show a part of the power spectrum where the second most conspicuous peak in the whole power spectrum can be found. The power is consistently concentrated at frequency $\sim 0.016 \frac{1}{\text { month }}$, which corresponds to $\sim 5.21$ years. Even though the peak can be understood in terms of a subharmonic of the main peak, we consider this periodicity to be due to the humps between the minima we have seen in Fig. 1. It can be considered as evidence that there are two populations of sunspots,as will be shown in a subsequent paper.

As stated above, we are only interested in short-term periodicities, and focus on these in the present analysis. In Fig. 4, we show the power spectrum obtained by

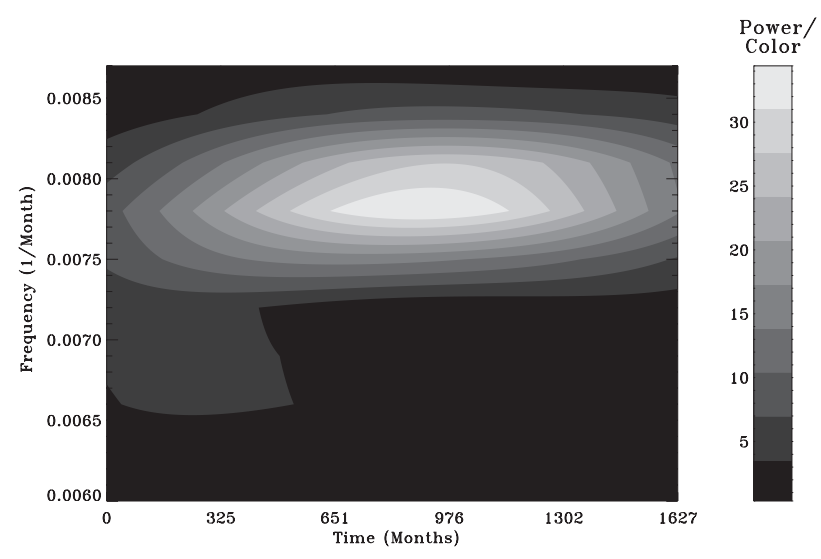

Fig. 2. The 2-dimensional power spectrum obtained by the wavelet analysis. The power spectrum is shown as a function of time in month and frequency in $\frac{1}{\text { month }}$. The data set covers 1,628 months from May in 1874 to December in 2009. The main peak occurs where the frequency is $\sim 0.0078$ $\frac{1}{\text { month }}$, whose period corresponds to $\sim 10.68$ years. 
the wavelet analysis in frequency ranges from 0.03 to $0.09 \frac{1}{\text { month }}$ to check whether the tendency of sunspots
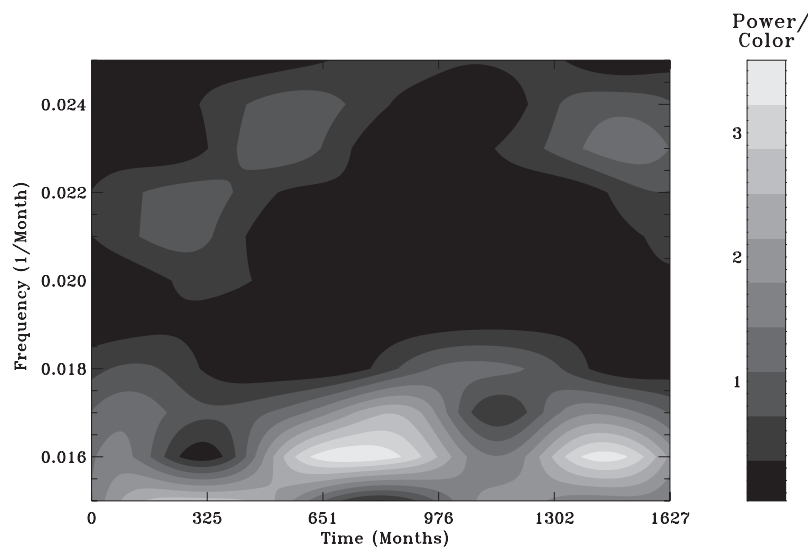

Fig. 3. A part of the whole power spectrum where the second most conspicuous peak can be found. The power is consistently concentrated at frequency $\approx 0.016 \frac{1}{\text { month }}$, which corresponds to $\sim 5.21$ years.

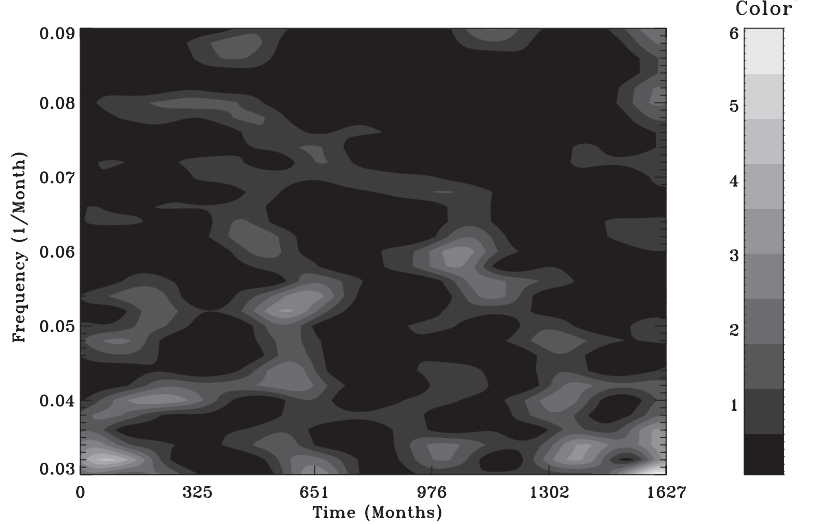

Fig. 4. A part of the whole power spectrum in frequency ranges from 0.03 to $0.09 \frac{1}{\text { month }}$. Any periodicities of $\sim 1.3(0.064), \sim 1.5(0.056)$, or $\sim 1.8$ (0.046) years $\left(\frac{1}{\text { month }}\right)$ can be hardly seen.

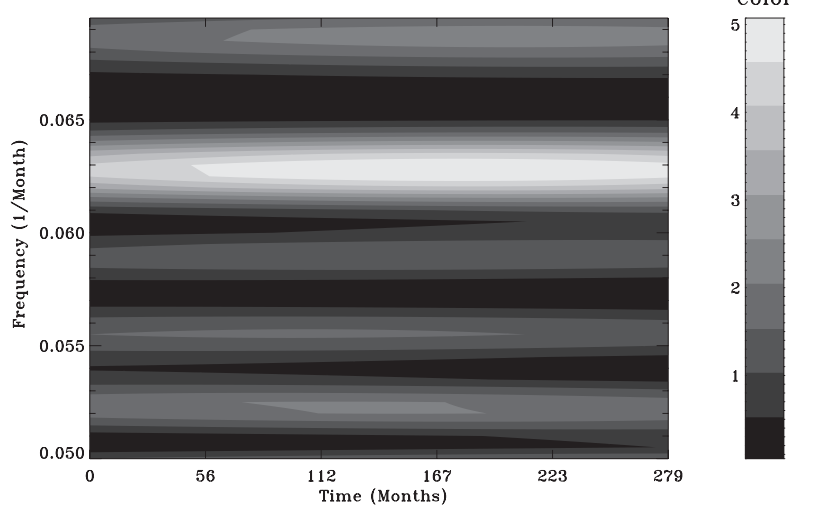

Fig. 5. Zoom-in the whole power spectrum in duration ranges from September in 1986 to December in 2009 (solar cycles 22 and 23) which overlap the data period when Howe et al. $(2000,2007)$ analyzed. to appear in limited latitudes indeed has biennial periodicities, which has been suggested as evidence of links between the changing structure of the sunspot zone and the tachocline rotation rate oscillations. This frequency range corresponds to one from 11 ( 0.91 years) to 33 months ( 2.75 years) in period. As shown in Fig. 4, however, the power appears as randomly distributed in almost the entire region of the power spectrum. In other words, we cannot find any periodicities of $\sim 1.3(0.064), \sim 1.5$ (0.056), or $\sim 1.8(0.046)$ years $\left(\frac{1}{\text { month }}\right)$, as claimed previously (Krivova\&Solanki 2002, Knaack et al. 2005, Ternullo 2010a). We, therefore, have to conclude that the oscillating component of $\sim 2$ year periodicity is insignificant and inconsistent. According to what we see in Figs. 3 and 4, the only reasonable short-term periodicity one can find in the latitudinal variation of sunspot positions is apparently $\sim 5.21$ years, but not $\sim 2$ years. Previously reported periodicities of $\sim 2$ years seem to be biased results due to small sunspots.

As a matter of fact, the 1.3 year periodicity detected by Howe et al. (2000) in the rotation rate at the base of the convection zone is still an open issue, in that Antia \& Basu (2000) and Basu \& Antia (2001), with a slightly different analysis of the same MDI and GONG data, reported no significant variations. Basu \& Antia (2001) saw only an insignificant signal. Moreover, the periodic signal disappears in the post-2001 data, even in Howe et al. (2007). In the later analysis by the original authors, the $\sim 1$ year periodicity was considered as variable. That is, even according to the original authors, the signal of $\sim 1.3$ year periodicity can be seen in the data set from 1995 to 2000 only, but cannot be seen in the data set from 2001 up to the near present. Even though we do not see any significant peaks around a frequency corresponding to $\sim 1.3$ years in the power spectrum in Fig. 4, we decide to zoom-in the power spectrum for the duration from September 1986 to December 2009 (solar cycles 22 and 23), whose duration overlaps the data period analyzed by Howe et al. (2000, 2007). If and only if we $d o$ see some similar temporal behaviors in the power distribution at frequency of $\sim 0.064$ $\frac{1}{\text { month }}$, which corresponds to the 16 month ( $\sim 1.3$ years $)$ period, we can still struggle to clean the power spectrum around that frequency to obtain a signal. As shown in Fig. 5 , unfortunately, we indeed find a consistent peak at frequency $\sim 0.063 \frac{1}{\text { month }}$ from $\sim 1990$ to 2009 , which is one of the other peaks of noise. In other words, there is no behavior seen as expected (i.e., only strong peaks from $\sim 1995$ to $\sim 2000$ and no peaks otherwise). Hence, even if what Howe et al. $(2000,2007)$ have found is a real feature, and even if the component at frequency $\sim 0.063$ is signifi- 
cant, we do not see any evidence whatsoever supporting its association in the latitudinal variation of the position of sunspots in view of COL.

\section{SUMMARY AND CONCLUSION}

The variation of area-weighted latitude of sunspots appearing during the period from 1874 to 2009 has been studied. We focus our investigation on short-term periodicities. We first determine the COL by averaging the latitude with the weight function in area. Then, we analyzed the time series of COL using the wavelet transform technique. We find that a periodicity of $\sim 5$ years is the second most dominant feature in the time series of COL. The power is consistently concentrated at frequency $\sim 0.016 \frac{1}{\text { month }}$. We consider this periodicity to be due to humps between the minima. We show the power spectrum in frequency ranges from 0.03 to $0.09 \frac{1}{\text { month }}$ to check whether the sunspot tendency to appear in limited latitudes indeed has biennial periodicities, which have been suggested as evidence of links between the changing structure of the sunspot zone and the tachocline rotation rate oscillations. As shown, however, we cannot find any periodicities of $\sim 1.3(0.064), \sim 1.5(0.056)$, or $\sim 1.8$ (0.046) years $\left(\frac{1}{\text { month }}\right)$, as claimed previously. We therefore have to conclude that the oscillating component of $\sim 2$ year periodicity is insignificant and inconsistent. Previously reported periodicities of $\sim 2$ years seem to be biased results due to small sunspots. Hence, even if what Howe et al. $(2000,2007)$ have found is a real feature, and even if the component at frequency $\sim 0.063$ is significant, we do not see any evidence whatsoever supporting its association in the latitudinal variation of the position of sunspots in view of COL.

\section{ACKNOWLEDGMENTS}

HYC is grateful to Sasha Kosovichev and Philip Scherrer for constructive discussions and hospitality while visiting Hansen Experimental Physics Laboratory, Stanford University where most of work has been done. This work was supported by a Research Grant funded by the Korea Aerospace Research Institute.

\section{REFERENCES}

Antalova A, Gnevyshev MN, Principal characteristics of the 11-year solar activity cycle, SvA, 9, 198-201 (1965).

Antalova A, Gnevyshev MN, Latitudinal distribution of sunspot areas during the period 1874-1976, CoSka, 11, 6393 (1983).

Antia HM, Basu S, Temporal variations of the rotation rate in the solar interior, ApJ, 541, 442-448 (2000). doi: 10.1086/ 309421

Basu S, Antia HM, A study of possible temporal and latitudinal variations in the properties of the solar tachocline, MNRAS, 324, 498-508 (2001). doi: 10.1046/j.13658711.2001.04364.x

Chang H-Y, A new method for north-south asymmetry of sun spot area, JASS, 24, 261-268 (2007). doi: 10.5140/ JASS.2007.24.4.261

Chang H-Y, Stochastic properties in North-South asymmetry of sunspot area, NewA, 13, 195-201 (2008). doi: 10.1016/j.newast.2007.08.007

Chang H-Y, Periodicity of North-South asymmetry of sunspot area revisited: cepstrum analysis, NewA, 14, 133138 (2009). doi: 10.1016/j.newast.2008.07.001

Cho I-H, Chang H-Y, Latitudinal distribution of sunspots revisited, JASS, 28,1-7 (2011). doi: 10.5140/JASS.2011.28. 1.00

Donner R, Thiel M, Scale-resolved phase coherence analysis of hemispheric sunspot activity: a new look at the North-South asymmetry, A\&A, 475, L33-L36 (2007). doi: 10.1051/0004-6361:20078672

Gleissberg W, Bemerkungen uber einen vermuteten extrasolaren Einfluss auf die Sonnentatigkeit, MitAG, 30, 151-153 (1971).

Goel A, Choudhuri AR, The hemispheric asymmetry of solar activity during the last century and the solar dynamo, RAA, 9, 115-126 (2009). doi: 10.1088/1674-4527/9/1/010

Grossmann A, Morlet J, Decomposition of Hardy functions into square integrable wavelets of constant shape, SIAM J Math Anal, 15, 723-736 (1984). doi: 10.1137/0515056

Hathaway DH, Nandy D, Wilson RM, Reichmann EJ, Evidence that a deep meridional flow sets the sunspot cycle period, ApJ, 589, 665-670 (2003). doi: 10.1086/374393

Howard R, LaBonte BJ, The sun is observed to be a torsional oscillator with a period of 11 years, ApJ, 239, L33-L36 (1980). doi: 10.1086/183286

Howe R, Christensen-Dalsgaard J, Hill F, Komm RW, Larsen RM, et al., Dynamic variations at the base of the solar convection zone, Sci, 287, 2456-2460 (2000). doi: 10.1126/science.287.5462.2456

Howe R, Christensen-Dalsgaard J, Hill F, Komm R, Schou J, et al., Temporal variations in solar rotation at the bottom of the convection zone: The current status, AdSpR, 40, 915-918 (2007). doi: 10.1016/j.asr.2006.12.047 
Javaraiah J, Long-term variations in the solar differential rotation, SoPh, 212, 23-49 (2003). doi: 10.1023/A:1022912430 585

Knaack R, Stenflo JO, Berdyugina SV, Evolution and rotation of large-scale photospheric magnetic fields of the Sun during cycles 21-23. Periodicities, north-south asymmetries and r-mode signatures, A\&A, 438, 1067-1082 (2005). doi: 10.1051/0004-6361:20042091

Krivova NA, Solanki SK, The 1.3-year and 156-day periodicities in sunspot data: wavelet analysis suggests a common origin, A\&A, 394, 701-706 (2002). doi: 10.1051/00046361:20021063

Le G-M, Wang J-L, Wavelet analysis of several important periodic properties in the relative sunspot numbers, ChJAA, 3, L391-L394 (2003). doi: 10.1088/1009-9271/3/5/391

Li KJ, Latitude migration of solar filaments, MNRAS, 405, 1040-1046 (2010). doi: 10.1111/j.1365-2966.2010.16508.x

Li KJ, Gao PX, Zhan LS, Synchronization of hemispheric sunspot activity revisited: wavelet transform analyses, ApJ, 691, L537-L540 (2009a). doi: 10.1088/0004-637X/691/1/ 537

Li KJ, Gao PX, Zhan LS, Synchronization of sunspot numbers and sunspot areas, SoPh, 255, 289-300 (2009b). doi: 10.1007/s11207-009-9328-7

Li KJ, Gao PX, Zhan LS, Shi XJ, Zhu WW, On the asynchronization of hemispheric high-latitude solar activity, MNRAS, 391, L34-L38 (2008). doi: 10.1111/j.17453933.2008.00551.x

Li KJ, Liang H-F, Feng W, Phase shifts of the paired wings of butterfly diagrams, RAA, 10, 1177-1185 (2010a). doi: 10.1088/1674-4527/10/11/008

Li KJ, Liang HF, Yun HS, Gu XM, Statistical behavior of sunspot groups on the solar disk, SoPh, 205, 361-370 (2002). doi: 10.1023/A:1014288424727

Li KJ, Liu XH, Gao PX, Zhan LS, The North-South asymmetry of filaments in solar cycles 16-21, NewA, 15, 346-350 (2010b). doi: 10.1016/j.newast.2009.09.009

Nagovitsyn YA, A nonlinear mathematical model for the solar cyclicity and prospects for reconstructing the solar activity in the past, AstL, 23, 742-748 (1997).

Pelt J, Brooke J, Pulkkinen PJ, Tuominen I, A new interpretation of the solar magnetic cycle, A\&A, 362, 1143-1150 (2000).

Pulkkinen PJ, Brooke J, Pelt J, Tuominen I, Long-term variation of sunspot latitudes, A\&A, 341, L43-L46 (1999).
Schwabe HS, Die Sonne, AN, 20, 283-286 (1843). doi: 10.1002/ asna.18430201706

Solanki SK, Wenzler T, Schmitt D, Moments of the latitudinal dependence of the sunspot cycle: a new diagnostic of dynamo models, A\&A, 483, 623-632 (2008). doi: 10.1051/0004-6361:20054282

Temmer M, Rybák J, Bendik P, Veronig A, Vogler F, et al., Hemispheric sunspot numbers Rn and Rs from 19452004: catalogue and N-S asymmetry analysis for solar cycles 18-23, A\&A, 447, 735-743 (2006). doi: 10.1051/00046361:20054060

Temmer M, Veronig A, Hanslmeier A, Hemispheric sunspot numbers Rn and Rs: catalogue and N-S asymmetry analysis, A\&A, 390, 707-712 (2002). doi: 10.1051/0004-6361: 20020758

Ternullo M, The butterfly diagram fine structure, SoPh, 240, 153-164 (2007a). doi: 10.1007/s11207-006-0261-8

Ternullo M, Looking inside the butterfly diagram, AN, 328, 1023-1032 (2007b). doi: 10.1002/asna.200710868

Ternullo M, The butterfly diagram internal structure, Ap\&SS, 328, 301-305 (2010a). doi: 10.1007/s10509-010-0270-9

Ternullo M, The butterfly diagram internal structure, MSAIS, 14, 202-205 (2010b).

Tuominen J, On the dependence of the systematic drift of sunspots in heliographic latitude on phase in the 22year cycle of the Sun. With 3 figures, ZA, 30, 261-274 (1952).

Waldmeier M, Der langeSonnenzyklus. Mit 3 Textabbildungen, ZA, 43, 149-160 (1957).

Waldmeier $\mathrm{M}$, The asymmetry of solar activity in the years 1959-1969, SoPh, 20, 332-344 (1971). doi: 10.1007/ BF00159763

Wolf R, Schreiben des Herrn Prof. R. Wolf, Directors der Sternwarte in Zurich an den Herausgeber, AN, 56, 355358 (1862). doi: 10.1002/asna.18620562303

Zolotova NV, Ponyavin DI, Phase asynchrony of the NorthSouth sunspot activity, A\&A, 449, L1-L4 (2006). doi: 10.1051/0004-6361:200600013

Zolotova NV, Ponyavin DI, Was the unusual solar cycle at the end of the XVIII century a result of phase asynchronization?, A\&A, 470, L17-L20 (2007). doi: 10.1051/00046361:20077681

Zolotova NV, Ponyavin DI, Marwan N, Kurths J, Long-term asymmetry in the wings of the butterfly diagram, A\&A, 503, 197-201 (2009). doi: 10.1051/0004-6361/200811430 